\title{
Controlling the dynamics of a bidimensional gel above and below its percolation transition
}

\author{
D. Orsi,${ }^{1}$ B. Ruta, ${ }^{2}$ Y. Chushkin, ${ }^{2}$ A. Pucci, ${ }^{3}$ G. Ruggeri, ${ }^{3}$ G. Baldi, ${ }^{4}$ T. Rimoldi, ${ }^{1}$ and L. Cristofolini ${ }^{1, *}$ \\ ${ }^{1}$ Department of Physics and Earth Sciences, University of Parma, Viale Usberti 7/A, I-43124 Parma, Italy \\ ${ }^{2}$ European Synchrotron Radiation Facility, Boîte Postale 220, F-38043 Grenoble, France \\ ${ }^{3}$ Department of Chemistry and Industrial Chemistry, University of Pisa, Via Risorgimento 35, I-56126 Pisa, Italy \\ ${ }^{4}$ IMEM-CNR Institute, Parma Science Park, I-43124 Parma, Italy
}

(Received 9 December 2013; published 25 April 2014)

\begin{abstract}
The morphology and the microscopic internal dynamics of a bidimensional gel formed by spontaneous aggregation of gold nanoparticles confined at the water surface are investigated by a suite of techniques, including grazing-incidence $\mathrm{x}$-ray photon correlation spectroscopy (GI-XPCS). The range of concentrations studied spans across the percolation transition for the formation of the gel. The dynamical features observed by GI-XPCS are interpreted in view of the results of microscopic imaging; an intrinsic link between the mechanical modulus and internal dynamics is demonstrated for all the concentrations. Our work presents an example of a transition from a stretched to a compressed correlation function actively controlled by quasistatically varying the relevant thermodynamic variable. Moreover, by applying a model proposed some time ago by Duri and Cipelletti [Europhys. Lett. 76, 972 (2006)] we are able to build a master curve for the shape parameter, whose scaling factor allows us to quantify a "long-time displacement length." This characteristic length is shown to converge, as the concentration is increased, to the "short-time localization length" determined by pseudo-Debye-Waller analysis of the initial contrast. Finally, the intrinsic dynamics of the system is then compared with that induced by means of a delicate mechanical perturbation applied to the interface.
\end{abstract}

DOI: 10.1103/PhysRevE.89.042308

PACS number(s): 82.70.Gg, 61.05.cf, 83.80.Kn, 68.18.Fg

\section{INTRODUCTION}

Films formed by nanoparticles confined at an interface, separating either liquid from air or two immiscible liquid phases, are extensively studied [1] due to their importance in a wide range of systems, from the stabilization of emulsions and of foams [2] to the formation of photonic or plasmonic membranes $[3,4]$. Films made by noble metal nanoparticles in particular have recently been shown to form mechanically robust, free-standing membranes $[5,6]$ which can be important for the fabrication of high-efficiency solar cells and displays.

Unlike the case of three-dimensional (3D) colloidal suspensions, which often can be described reasonably well using DerjaguinLandauVerwey-Overbeek theory, the description of particles at interfaces requires the consideration of interfacial deformations as well as interfacial thermal fluctuations, as complex intercolloidal forces act within these 2D assemblies, which also depend on the contact angle of the individual colloids, with possible long-ranging effects. Moreover, hydrodynamic coupling between the 2D films and the bulk of the surrounding fluids complicates the measurement and the modeling of surface dynamic properties. For all these reasons, the theoretical comprehension of interfacial colloidal systems still represents an outstanding problem in the modern theory of colloidal interactions [7].

Langmuir monolayers of colloids at the air-water interface constitute a model system commonly used to investigate the structure and properties of such interfacial systems, recently applied also to the study of films of gold nanoparticles [8]. We remark that accurate structural and mechanical characterizations, with a quasistatically increase of the concentration, are peculiar to the 2D geometry; a comparable analysis

\footnotetext{
*luigi.cristofolini@unipr.it
}

on 3D systems would be much more difficult. Interfacial stability may become an issue when the concentration is increased. Structural investigations by grazing-incidence $x$-ray scattering have been employed to elucidate the structure of deposited Langmuir-Blodgett [9] and unsupported Langmuir monolayers [10]. In favorable cases, the contact angle of individual particles could be estimated by accurate modeling of the x-ray reflectivity curve $[11,12]$.

In these films, high-concentration regimes may present temporal and spatial dynamical heterogeneities, in conjunction with the arising of slow dynamics often leading to dynamical arrest and to aging phenomena. Such heterogeneities reflect the complexity of the structural arrangements and interactions among the colloids. As a matter of fact, dynamical heterogeneities of different kind are the fingerprints of the different models that over the years have been put forward to describe dynamical arrest. Their detailed quantification is therefore a crucial step in understanding the dynamics of systems approaching the arrested state [13].

This task can be accomplished by measuring high-order correlation functions, e.g., to characterize the non-Gaussian dynamics of arrested systems [14,15]. In previous work, we have been able to identify heterogeneities by grazing-incidence x-ray photon correlation spectroscopy (GI-XPCS) [16] in a Langmuir polymeric monolayer [17] and in the highly compressed phase of a $2 \mathrm{D}$ gel generated as a Langmuir monolayer of gold nanoparticles [18]. In the former case, heterogeneous dynamics could be observed clearly in the photoinduced fluid phase of the polymer, while in the latter case, heterogeneity was found to be growing with increasing surface compression, and the quality of the data was good enough to allow the experimental determinations of the fourthorder temporal correlation function in an arrested system by GI-XPCS. 
The morphology of the same system has been investigated in detail by microscopy techniques, revealing a complex structure with features on a hierarchy of different sizes following a Lévy distribution [19]. The evolution of the statistical properties of the structured network, as a function of the externally controlled density, can be related to the corresponding evolution of the mechanical properties. In the incubation time, while the film is left at rest, individual nanoparticles (NPs) diffuse on the surface and aggregate, presumably driven by hydrophobic interaction between the dodecanethiol coatings. This results in the formation of small disklike aggregates. During compression, we can identify three steps leading to the gel formation: A first step occurs when disks of sufficient size aggregate because of their mutual attraction, which is due to the indentation effects that those disks exert on the water surface. In this regime, as soon as an elongated aggregate is formed, e.g., by sticking together of two disks, the aggregation is likely to proceed tip to tip in a fashion similar to that reported in [20], resulting in the formation of quasi-1D structures, which can reach a typical length of a few microns. Once those acicular aggregates are formed, a second step occurs with monolayer compression, resulting in the growth of a branched structure, finally yielding to the onset of the infinite percolative cluster, which is related to the building of the mechanical elastic modulus. The percolating transition occurs around $\Phi_{c}=31.9 \%$ [21]. The third step of formation of the network is final compaction of this structure.

It is well known that fluctuation dynamics are strictly related to mechanical moduli, via generalized fluctuation-dissipation relations [22].This has been extensively exploited for the characterization of the rheology of interfacial layers: the measurement of their mechanical properties is a delicate task that can be performed by a number of techniques, which can be broadly divided into active and passive ones. In the former case, a deformation is applied from outside, as in the oscillating barriers technique [23] and in interfacial shear rheology (ISR), in which a magnetized needle is used to induce shear deformation [24,25]. In the latter case, mechanical properties are deduced from the spontaneous fluctuations recorded, e.g., by dynamic light scattering or by XPCS experiments [17,26,27]. A priori, the combination of the results of such measurements to yield a comprehensive picture is complicated by the different space scales involved, spanning from the centimeter scale for the ISR down to fractions of a micron in the case of XPCS. However, in most cases, those macroscopic and microscopic quantities have been found to agree, as, e.g., in floating polymeric layers [17,26]. A counterexample of this is found even in simple phospholipid monolayers; their macroscopically liquid and mainly viscous phase may exhibit a complex and mainly elastic response when probed on the micron scale [28].

In the present paper we report a detailed study of the spontaneous fluctuation dynamics of a Langmuir monolayer of gold nanoparticles floating on the water surface, together with the relation to its structure and to its mechanical response. This is done by exploiting a peculiarity of the Langmuir technique, varying the surface concentration from well below to well above the threshold for the formation of a percolative cluster. The sample, its preparation, and the experimental methods used are described in detail in Sec. II. We start with a characterization of the system by different microscopy techniques such as in situ epimicroscopy of the Langmuir monolayer and scanning electron microscope (SEM) imaging of the monolayers transferred onto a solid substrate; next, we report on the measurement of the mechanical moduli of the film (Sec. III A). Then, by means of GI-XPCS we focus on the spontaneous fluctuation dynamics and the connections to mechanical moduli; we investigate the evolution while crossing the percolation threshold (Sec. III B). We discuss the changes in the dynamics and their connection with structure and mechanical moduli in light of different theories. Finally, we report on the effects that an external mechanical perturbation exerts on the fluctuation dynamics of the system, with particular regard to transient states (Sec. III C).

\section{SAMPLE AND METHODS}

\section{A. Sample}

Nanoparticles were synthesized as follows, by the twophase Brust method [29,30]. In brief, $0.400 \mathrm{~g}(1.02 \mathrm{mmol})$ of hydrogen tetrachloro-aurate (III) trihydrate were dissolved in $30 \mathrm{ml}$ of deionized water. The solution was then shaken in a separatory funnel with $80 \mathrm{ml}$ of toluene solution containing $2.00 \mathrm{~g}(3.56 \mathrm{mmol})$ of tetra- $n$-octylammonium bromide (TOAB). The toluene phase was then recovered and combined with $0.018 \mathrm{~mL}(0.015 \mathrm{~g}, 0.07 \mathrm{mmol})$ of dodecyl mercaptan. A freshly prepared aqueous solution of sodium borohydride $(25 \mathrm{ml}, 0.386 \mathrm{~g})$ was slowly added under vigorous stirring. After further stirring for $3 \mathrm{~h}$, the organic phase was separated, concentrated to $10 \mathrm{ml}$, and mixed with $70 \mathrm{ml}$ of ethanol. The mixture was cooled overnight at $-20^{\circ} \mathrm{C}$ and the dark precipitate then recovered by filtration. The crude product was then purified by soxhlet extraction with acetone as cleansing solvent to remove all the unbound free thiol and residual TOAB impurities, thus making the gold NPs (GNPs) fully soluble in organic solvents. The average diameter of the GNPs was estimated to be $8 \mathrm{~nm}$ by dynamic light scattering (DLS) analysis with a $6 \mathrm{~nm}$ diameter gold nucleus [transmission electron microscopy (TEM) analysis] [31].

The Langmuir monolayer was prepared as follows: nanoparticles were dispersed in chloroform, $50 \mathrm{mg}$ in $10 \mathrm{ml}$, thus obtaining a $5 \mathrm{mg} / \mathrm{ml}$ suspension used for long-term storage in a refrigerator. Before each experiment, a small batch of less concentrated suspension was prepared by dilution of the stock solution in hexane, to reach the final concentration of $0.2 \mathrm{mg} / \mathrm{ml}$. This suspension was slowly spread at the air/water interface using a $100 \mu \mathrm{l}$ Hamilton syringe; the tip of the syringe's needle was kept in contact with the water surface during the whole process. A total volume of $2.5 \mathrm{ml}$ was spread on the Langmuir trough used in GI-XPCS experiments: a spreading time of 2 min was used for the spreading of each syringe. A 20 min waiting time was scheduled to allow solvent evaporation and to achieve the equilibration of the sample. During the experiments the water subphase was kept at constant temperature $\left(18^{\circ} \mathrm{C}\right)$, by means of water circulation from a Lauda thermal bath through the Langmuir trough's basement. 


\section{B. Rheology measurements}

The mechanical response of the 2D system was completely characterized, by measuring both the compression $(\varepsilon)$ and shear $(G)$ complex moduli as functions of surface concentration. This was accomplished by using two complementary techniques operating — on the macroscopic scale_-on the same temporal scale: the oscillating barriers and the oscillating needle techniques.

The oscillating barriers technique exploits the difference in the surface pressure measured by perpendicular Wilhelmy plates when a dense Langmuir film is subject to uniaxial compression [23,32].The experimental setup consisted of a Langmuir trough (custom made, size $10 \times 40 \mathrm{~cm}^{2}$, with $\mathrm{KSV}$ Nima mechanics) equipped with two identical Wilhelmy balances, which monitor the evolution of the surface tension (and hence the phase and amplitude of their oscillations $\Delta \Pi_{\|}$ and $\Delta \Pi_{\perp}$ ) while the area available to the film is oscillated by the synchronized movement of the two barriers, operated at atypical frequency of $0.5 \mathrm{~Hz}$.

From the area $A_{0}$, its oscillation $\Delta A$, and the phase lag $\vartheta$ we obtain the complex mechanical moduli:

$$
\begin{gathered}
G=G^{\prime}+i G^{\prime \prime}=A_{0}\left(\frac{\Delta \Pi_{\|}}{\Delta A}-\frac{\Delta \Pi_{\perp}}{\Delta A}\right) e^{i \vartheta}, \\
\varepsilon=\varepsilon^{\prime}+i \varepsilon^{\prime \prime}=A_{0}\left(\frac{\Delta \Pi_{\|}}{\Delta A}+\frac{\Delta \Pi_{\perp}}{\Delta A}\right) e^{i \vartheta} .
\end{gathered}
$$

This analysis assumes linearity in the response of the system to the external perturbation. This assumption was observed to hold in the diluted regimes, while at concentrations significantly higher than the onset of percolation, we observed important nonlinearities in the compression response even for the smallest amplitude of perturbation that was applicable. For this reason the oscillating needle technique, which imposes a pure shear perturbation, was employed to characterize the latter regime. The oscillating needle rheometer employed is an adapted version of the instrument proposed years ago by Fuller and co-workers [24] and is described in detail in [25]. In brief, a stainless steel needle ( $1 \mathrm{~cm}$ long, $0.3 \mathrm{~mm}$ radius) magnetized to saturation is incorporated in the Langmuir film and oscillates under the action of a suitable magnetic field gradient whose equilibrium position is oscillated in a sinusoidal manner. A fast CCD camera measures the amplitude and the phase of the oscillations of the needle. The mechanical shear modulus $G$ is then obtained as follows:

$$
G=G^{\prime}+i G^{\prime \prime}=\frac{\sigma}{\gamma} e^{i \vartheta}
$$

where $\sigma$ is the stress exerted by the needle, $\gamma$ is the resulting strain, and $\vartheta$ is the phase difference between the oscillations of the magnetic field and of the needle.

\section{GI-XPCS measurements}

Multispeckle GI-XPCS experiments were performed at the beamline ID10 of the European Synchrotron Radiation Facility (ESRF) in Grenoble, France. An incident x-ray beam with an energy of $8 \mathrm{keV}(\lambda=0.155 \mathrm{~nm})$ was selected from the undulator radiation by a $\mathrm{Si}(111)$ pseudo-channelcut monochromator scattering in vertical geometry (energy bandwidth $\left.\Delta E / E \sim 10^{-4}\right)$. The beam was then focused with Be compound lenses while higher harmonics were suppressed by two Si mirrors. The spatially coherent part of the incoming radiation was selected by using roller-blade slits opened to $10 \times 10 \mu \mathrm{m}^{2}$, placed $\sim 0.18 \mathrm{~m}$ upstream of the sample. The parasitic scattering produced by the beam-defining slits was removed by carefully adjusting a set of guard slits a few centimeters upstream of the sample. The resulting incident flux on the sample was $10^{9}$ photons/s/100 mA. The beam was reflected to impinge on the liquid surface at a grazing incident angle of $0.137^{\circ}$, which is about $90 \%$ of the critical angle for total reflection on the water surface at this photon energy.

A custom-made Langmuir trough with a single moving barrier (maximum surface $418 \times 170 \mathrm{~mm}^{2}$ ) was installed on the sample diffractometer. The trough was mounted on an active antivibration support and provided with a plastic enclosure, under which a helium atmosphere was created in order to minimize parasitic scattering from air and at the same time reduce risks of beam-induced damage. KSV-Nima Wilhelmy balance and electronics were used to control the trough and measure surface pressure and the trough's area during GI-XPCS measurements. The mechanical perturbation of the film was provided by a 4-cm-long magnetized needle that was put in oscillation by two magnets inserted for this purpose. The magnets were suspended parallel to the water surface by means of a plastic support, with their dipoles mutually perpendicular. One of them was permanent, needed to define the equilibrium position and orientation of the needle, while the second, an externally controlled electromagnet, was used to rotate the needle, thus providing the required perturbation to the film.

Two-dimensional $\mathrm{x}$-ray scattering speckle patterns were recorded by using a photon-counting area detector (Medipix, $256 \times 256$ pixels, $55 \mu \mathrm{m}$ pixel size [33]) placed at a distance of $3.3 \mathrm{~m}$ from the sample. The exposure time of the Medipix detector was chosen to be long enough to warrant a reasonable signal-to-noise ratio, $10-100 \mathrm{~ms}$ in our case. To avoid unnecessary irradiation of the sample, a fast shutter, synchronized with the area detector, was placed upstream of the sample. Sets of up to 20000 images were collected for different sample concentrations with different exposure times. The intensity-time correlation functions were calculated from the series of two-dimensional images by software.

\section{RESULTS AND DISCUSSION}

\section{A. Morphology and mechanical response}

Exploiting the peculiarity of the 2D geometry of a Langmuir experiment, we performed experiments covering a wide range of surface concentrations $\Phi$. In our system, as $\Phi$ is increased, a 2D network of gold nanoparticles is formed. The process of formation of the network has several similarities with a percolation transition, as has been thoroughly characterized in a microscopy experiment [21]. A sample of the resulting network, imaged by an inverted microscope, acquired with a $50 \times$ objective, is shown in Fig. 1(a).

Figure 1(b) shows the surface pressure-concentration П- $\Phi$ isotherm recorded during the GI-XPCS experiment. The determination of the surface concentration $\Phi$ is very important for the following discussion, and therefore it has 

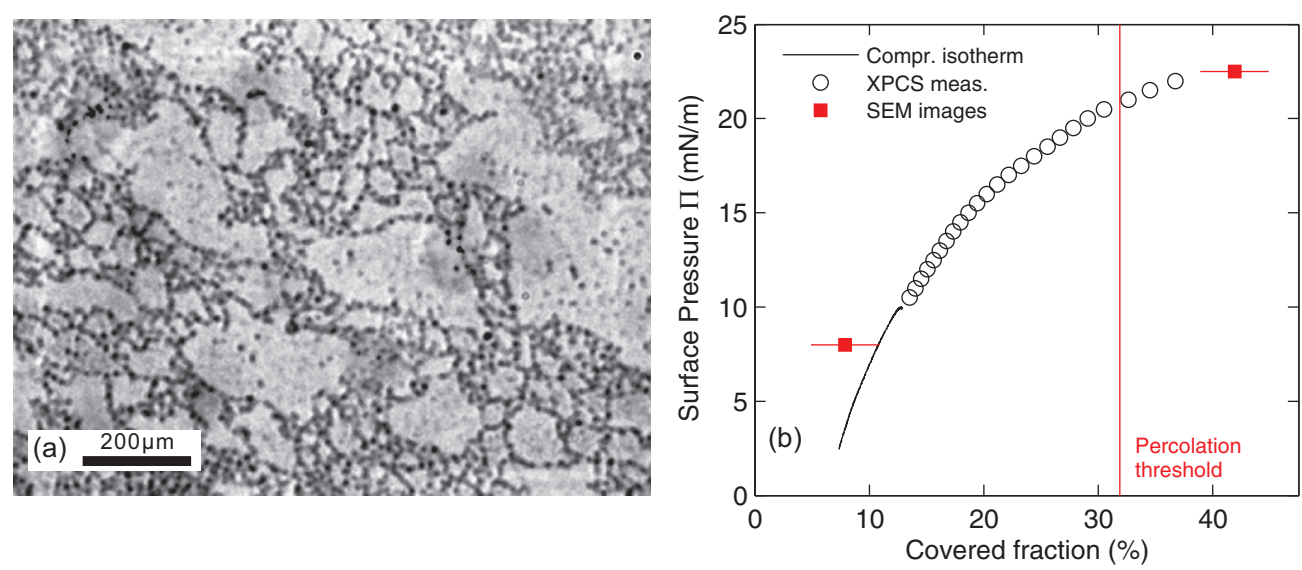

FIG. 1. (Color online) At each surface pressure П, the concentration of gold nanoparticles at the surface is monitored by imaging. (a) Image of the GNP network at $\Phi=29 \%$, taken using an inverted microscope with a $50 \times$ objective. (b) Surface-pressure-concentration isotherm measured during the GI-XPCS experiment. GI-XPCS measurements were performed at the concentrations indicated by the empty circles. As a double check, $\Phi$ was also directly measured by SEM imaging on two samples transferred onto solid silicon substrate (red squares).

been cross-checked by employing different techniques: In our laboratory, besides calculating $\Phi$ from the spread amount, as usual in Langmuir experiments, we also performed in situ epimicroscopy and Brewster angle microscopy on the film floating at the air/water interface, in addition to SEM imaging of samples of film transferred onto the silicon substrate. The estimates of $\Phi$ arising from these investigation are all consistent with that calculated from the spread amount of nanoparticles [19,21].

For the GI-XPCS experiments reported here, the estimate from the spread amount has been cross checked ex post facto by SEM imaging of samples of film transferred onto a silicon substrate at selected values of $\Pi$. The resultsreported in Fig. 1(b) - show good agreement between the two determinations.

The evolution of the mechanical response above and below the percolation transition has been measured using the techniques discussed in the experimental section; results are reported in Fig. 2. It is reasonable to postulate that, at very dilute concentrations, a viscous (dissipative) regime should exist, which, however, may correspond to extremely low values of the moduli, well below the sensitivity limits of the experimental techniques. In the range relevant to our study, both shear $(G)$ and compression $(\varepsilon)$ moduli are mainly real, indicating an elastic response, in agreement with previous results on the same system [19]. There is an important difference, however, between the shear and compression responses. As shown in Fig. 2(a), $G$ presents a transition between two different regimes: for $\Phi<25 \%$ it has a very small value (below $0.2 \mathrm{mN} / \mathrm{m}$, close to the sensitivity limit of the technique), while as the percolation threshold is approached, $G$ starts to increase, reaching a saturation value of the order of $1-2 \mathrm{mN} / \mathrm{m}$, above which it enters a nonlinear regime, indicative of the intrinsic brittleness of this structure. On the contrary, as shown in Fig. 2(b), the compression modulus $\varepsilon$ stays constant at all the concentrations below the threshold and nonlinear effects dominate above this concentration.

The elastic nature of the mechanical response found below $\Phi_{c}$ has to be attributed to some kind of long-range repulsion that dominates the dynamics in this diluted regime.
Its origin could be electrostatic or could be due to the organic dodecanethiol molecules coating the NPs and providing the thermodynamic stabilization of NPs in solution through steric repulsion.

\section{B. Intrinsic dynamics probed by GI-XPCS}

The dynamics of the GNP network has been measured by recording the $\mathrm{x}$-ray intensity scattered from the

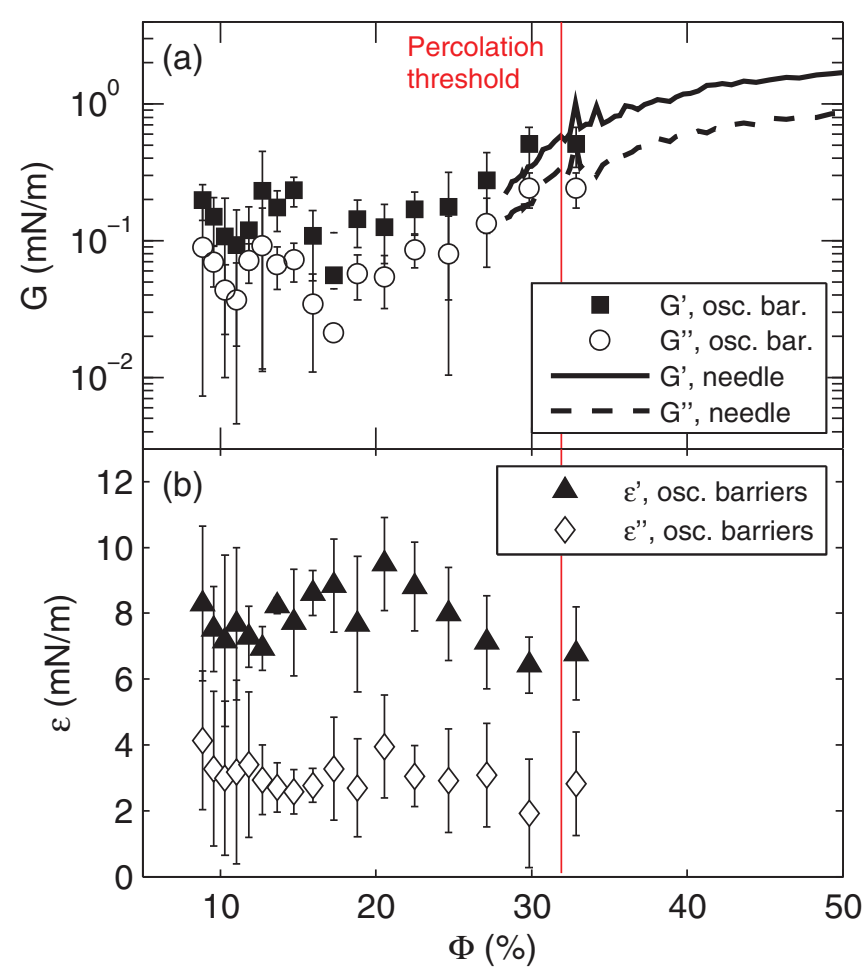

FIG. 2. (Color online) $\Phi$ dependence of the mechanical properties of the GNP network during its formation process. (a) The network shows mainly an elastic shear modulus, which increases with $\Phi$. (b) The compressibility stays constant up to the percolation threshold (vertical line). 
sample in the grazing-incidence diffraction experiment described in the experimental section, at several values of $\Phi$ and in correspondence of different values of the components of the scattering vector in the directions parallel $\left(q_{\|}\right)$and perpendicular $\left(q_{\perp}\right)$ to the air/water interface.

The pixels of the 2D detector were divided into square groups, each of which is labeled by its scattering vector components $\left(q_{\|}, q_{\perp}\right)$. From the scattered intensity measured by each group of pixels as a function of time we calculate the intensity autocorrelation function

$$
g^{(2)}\left(q_{\|}, q_{\perp} ; t\right)=\frac{\left\langle I\left(t_{0}\right) I\left(t_{0}+t\right)\right\rangle_{t_{0}}}{\left\langle I\left(t_{0}\right)\right\rangle_{t_{0}}^{2}}
$$

In soft-matter experiments, the correlation functions are commonly described using the empirical Kohlrausch-WilliamWatts (KWW) exponential

$$
g^{(2)}\left(q_{\|}, q_{\perp} ; t\right)=A+\beta e^{-2(t / \tau)^{\gamma}},
$$

where $\tau$ is the relaxation time of the dynamics, $\beta$ is the contrast, and $\gamma$, called the shape parameter, is connected to the kind of dynamics that characterizes the system, together with the dependence of $\tau$ on $q$. We briefly recall here that, in the case of Brownian dynamics, $\tau \propto q^{-2}$ and $\gamma=1$; pure ballistic motion shows $\tau \propto q^{-1}$ and $\gamma=2$ [34]; while more complex systems display some intermediate behavior, e.g., many arrested systems show a compressed shape often with $\tau \propto q^{-1}$.

Figure 3 reports data and fit models for correlation functions measured at $\Phi=15 \%$. The initial contrast of the correlation functions shows a clear dependence on $q$. When additional relaxation processes faster than the exposure time are present, they cause a sort of "blurring" of the speckle pattern. This implies a reduction of the contrast $\beta$ with respect to its theoretical value $\beta_{0}$; this reduction depends on $q$ following

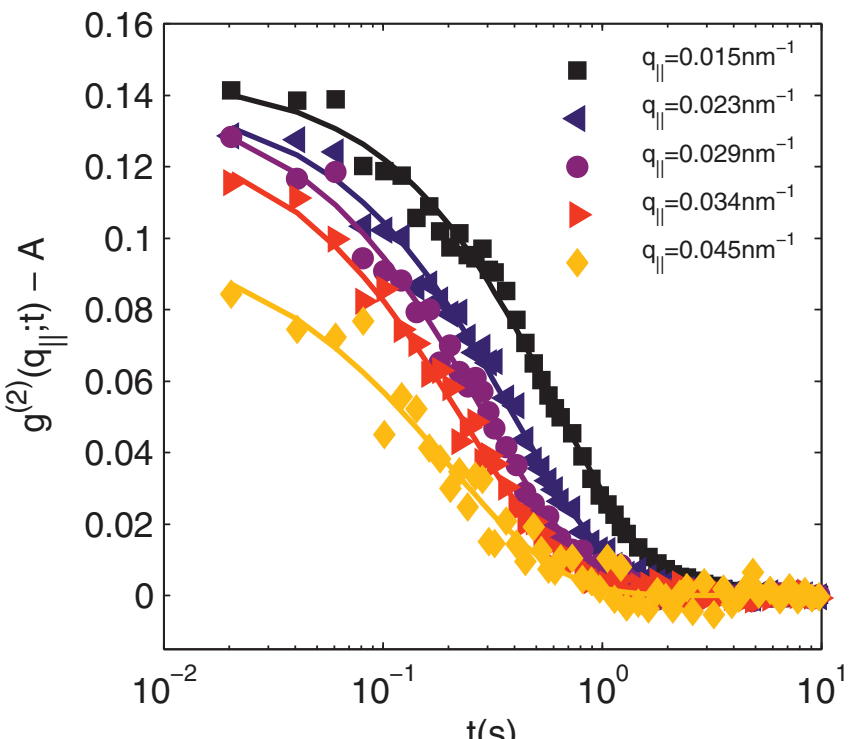

$t(s)$

FIG. 3. (Color online) correlation functions decay as KWW exponentials. Here, the correlation functions measured at $\Phi=15 \%$ at several values of $q$ are shown. Continuous lines are fits to the KWW phenomenological law. a pseudo-Debye-Waller decay [27] ruled by the localization length $r_{\text {loc }}=\sqrt{\left\langle u^{2}\right\rangle}$ :

$$
\beta=\beta_{0} \exp \left(-\frac{q_{\|}^{2}\left\langle u^{2}\right\rangle}{3}\right) .
$$

This is clearly the case for our data, as inspection of Fig. 3 shows that the initial contrast is well below the theoretical value, which in our experimental geometry is approximately $\beta_{0}=0.2$. In Fig. 4(a) we report the $q$ dependence of $\beta$ together with the fits to Eq. (6). By this analysis, the localization length $r_{\text {loc }}$ can be extracted, as reported in Fig. 4(b). At low concentration, the value of $r_{\text {loc }}$ decreases linearly with increasing $\Phi$, reaching a value compatible with the particle's diameter $(8 \mathrm{~nm})$ at about $22 \%$; then this decrease saturates at higher concentrations. The linear decrease is marked by the black line in Fig. 4(b).

The initial decrease of $r_{\text {loc }}$ may be interpreted as if the fast single-nanoparticle motion becomes more and more hindered by the spatial constraints imposed by neighbors, while interfacial aggregates are formed. Then the saturation of $r_{\text {loc }}$ above $22 \%$ is consistent with the idea that at this stage individual NPs are localized within the gel branches, confined by nearest neighbors [21]. Further increasing concentration affects the slow dynamics of the whole film, but not the localization length due to fast motion of single NPs.

In Fig. 5(a) we report the values of $\tau$ obtained from the fits to the KWW relation of the correlation functions measured at $\Phi=15 \%$. In agreement with our previous investigations [18] we find that $\tau$ depends only on the parallel component of the scattering vector $q_{\|}$, which varies on the horizontal axis in the figure, and not on the perpendicular component $q_{\perp}$, thus confirming that the dynamics is confined at the air/water interface. Therefore, in all the subsequent analysis, we averaged the results along $q_{\perp}$ to improve the statistics. We also find that $\tau$ scales always as $\tau \propto q_{\|}^{-n}$ [Fig. 5(b)] with $n$ of the order of 1 [Fig. 8(b) below], a feature common to many arrested systems.

Notably, the shape exponent $\gamma$, shown in Fig. 5(c), varies as a function of $q_{\|}$and of the concentration $\Phi$. In the range covered by the present study we are able to document a transition from a stretched $(\gamma<1)$ to a strongly compressed $(\gamma>1.6)$ shape as the concentration is increased and $q_{\|}$is decreased.

The contour line marks the threshold value of $\gamma=1$, corresponding to simple exponential decay, while the dashed line indicates the threshold concentration for the onset of the percolative cluster. The observed change in shape is a signature of a change in the physical mechanism responsible for the underlying dynamics. While a stretched shape would suggest a distribution of Brownian diffusors, with a heterogeneous distribution of relaxation times, the transition to a compressed shape, accompanied by $n \approx 1$ is a signature of an arrested system. Similar stretched-compressed dynamical crossovers have been also reported in a few different out-of-equilibrium materials such as nanoparticles in a glass-former matrix [35], metallic glasses [36], and, very recently, in Laponite suspensions by Angelini et al. [37]. In the last, the authors observed $\gamma \approx 0.75$ in an aged Laponite suspension $\left(C_{w}=3.0 \%\right)$, while 

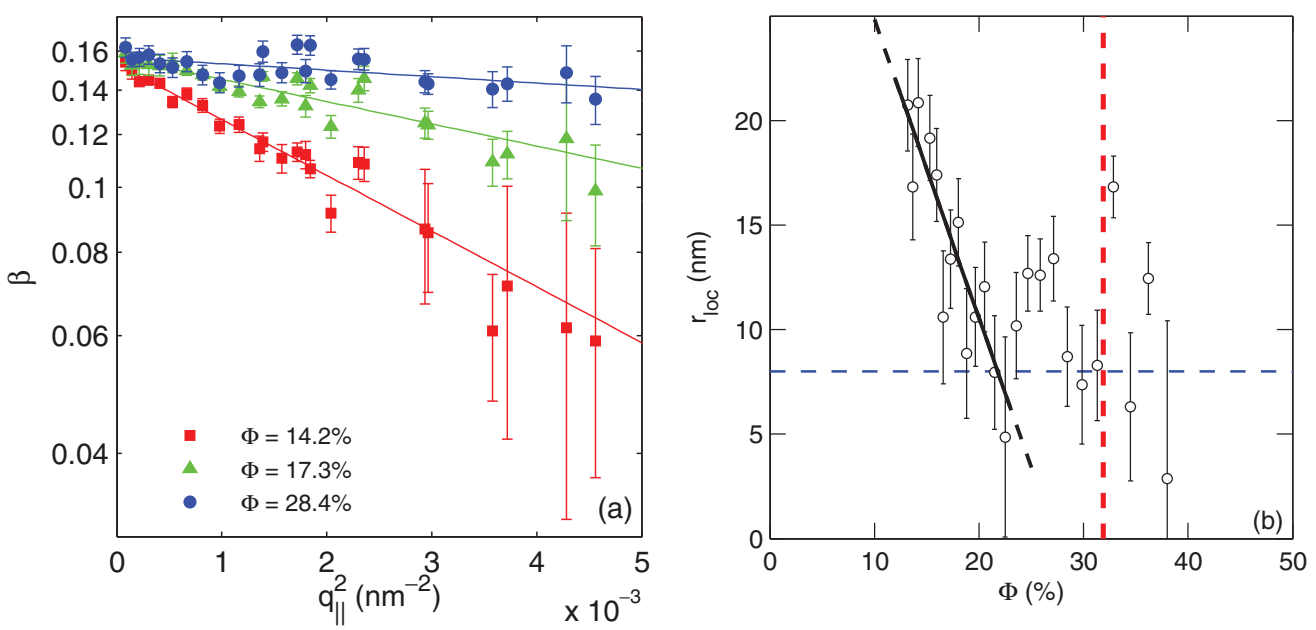

FIG. 4. (Color online) The contrast decays with $q_{\|}$following a Debye-Waller dependence. (a) Data for three different concentrations are plotted on a semilogarithmic scale as a function of the squared scattering vector. Lines are fits to Eq. (6). (b) Localization length $r_{\text {loc }}$ as a function of concentration: with increasing $\Phi$ it decreases to reach a value comparable with the size of an individual nanoparticle (8 nm, horizontal dashed blue line). The vertical red line represents the percolation threshold.

rejuvenation by means of mechanical perturbation seems to induce a more compressed shape $(\gamma=1.5$ at low $q$, decaying to 1.25 as $q$ increases).

Figure 6(a) represents a different way of recasting the data of Fig. 5(c), highlighting that, at a given $q_{\|}$, it is possible to control the shape of the correlation functions from stretched to compressed, at will, just by varying the surface concentration, which is achieved simply by moving the barriers of the Langmuir trough. In detail, at every $q_{\|}$value, we observe a linear relation of $\Phi$ and $\gamma$, with the same slope and varying intercept, whose dependence on $q_{\|}$is depicted in Fig. 6(b).
The complex dynamics of our system are not trivial to interpret on a microscopic scale. A compressed relaxation is predicted within a detailed model proposed by Bouchaud and Pitard [38] for the dynamics in an elastic solid. Within this model, randomly appearing dipolar stresses generate a field of strains in the network of the elastic gel. It is then assumed that the dynamics of the diffusors is determined by the relaxation of such local strains, leading to the compressed shape of the correlation functions. This detailed model, however, puts strict constraints on the $q$ dependence of $\gamma$ (predicting two regimes, $\gamma=1.5$ for small $q$ and $\gamma=1.25$ for large $q$ ), which do not agree with our data.
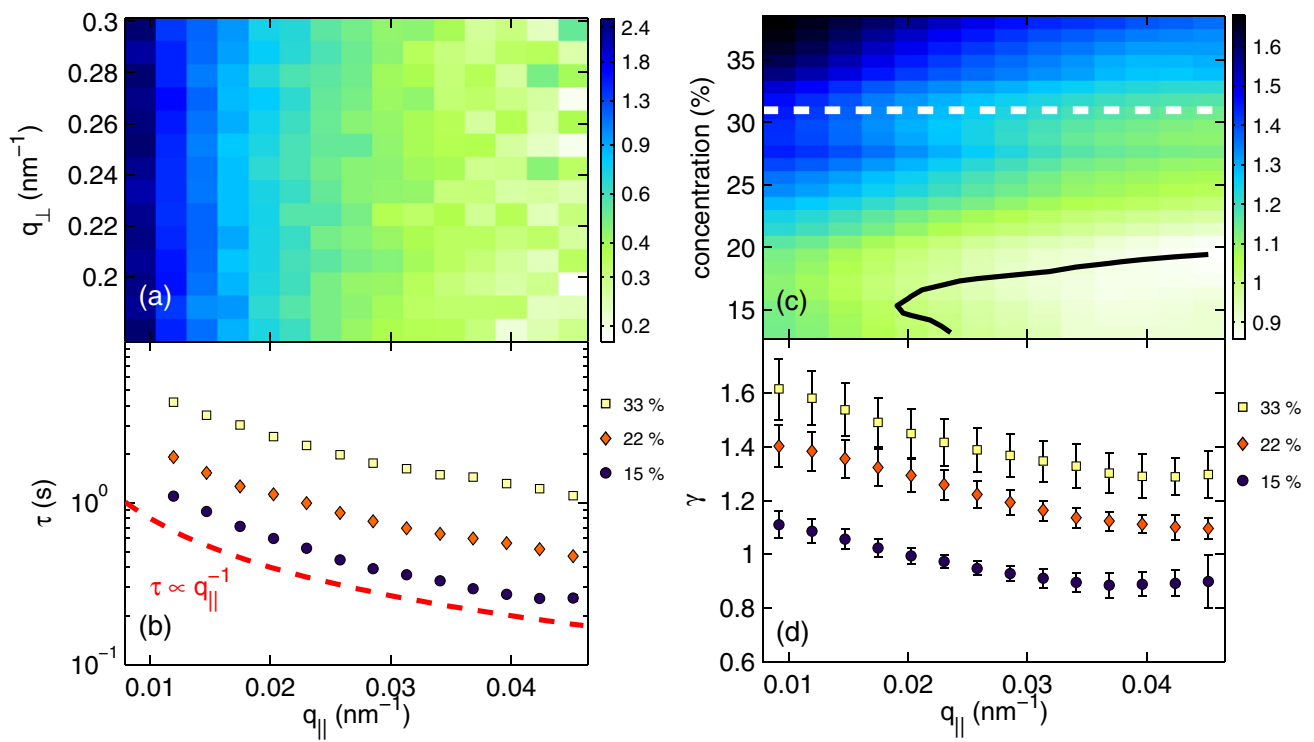

FIG. 5. (Color online) Both the relaxation time $\tau$ and shape exponent $\gamma$ decrease at large $q_{\|}$. (a) Map of relaxation time $\tau$ as a function of the two components of the scattering vector, $q_{\|}$and $q_{\perp}$, for $\Phi=15 \%$. Values of the color scale are in seconds. (b) Detailed dependence of $\tau$ on $q_{\|}$measured at different concentrations $(\Phi=15 \%, 22 \%, 33 \%)$. The $\tau \propto q_{\|}^{-1}$ behavior is also shown for comparison (dashed line). (c) Shape parameter $\gamma$ as a function of $q_{\|}$and of $\Phi$. The black line represents the contour of $\gamma=1$, i.e., of simple exponential decay, while the white dashed horizontal line indicates the critical concentration (see text). (d) Detailed $q_{\|}$dependence of $\gamma$ for the same concentrations as in (b) $(\Phi=15 \%, 22 \%, 33 \%)$. 

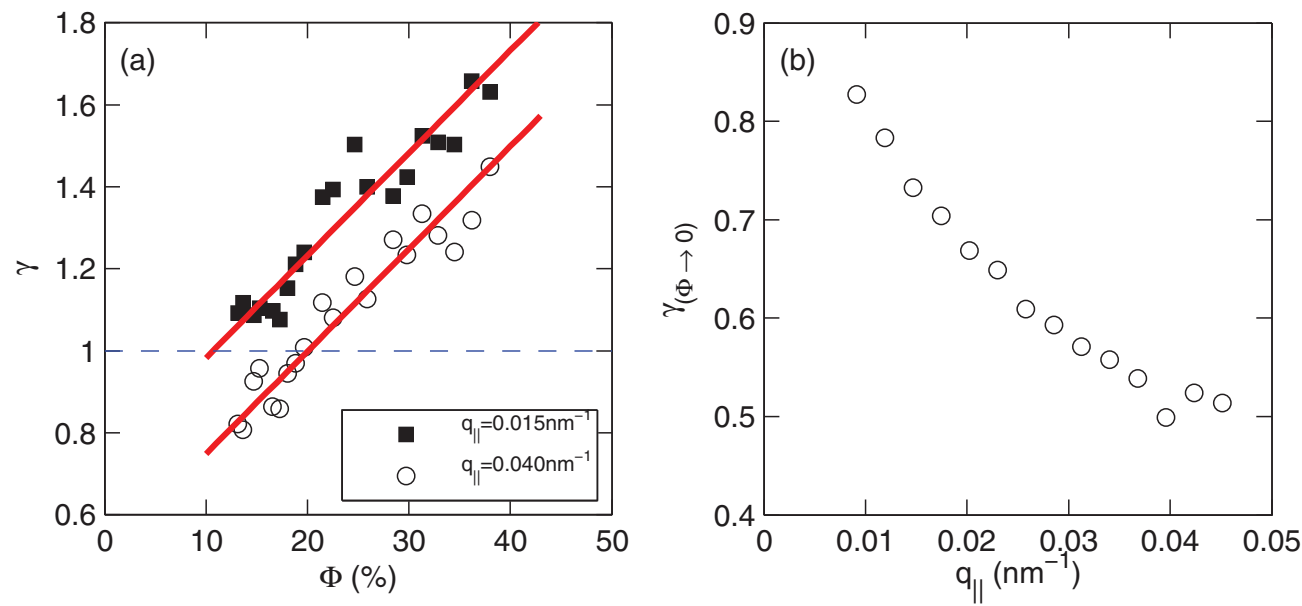

FIG. 6. (Color online) (a) The dependence of $\gamma$ on $\Phi$ is linear in the $q_{\|}$range explored. Continuous lines are linear fits. The dashed line indicates the simple exponential shape $\left(\gamma=1\right.$ ). (b) The extrapolation of $\gamma$ at $\Phi \rightarrow 0$ decreases with increasing $q_{\|}$.

A more phenomenological model was put forward by Duri and Cipelletti some years ago [39]. Those authors attribute the compressed shape for the relaxation found in DLS and XPCS experiments to rare, intermittent rearrangements. They deduce a variation of the shape from compressed with $\gamma=1.5$ to simple exponential as a function of the reduced scattering vector, obtained by scaling $q$ by a typical length $\delta$ characterizing the displacement. In this case, the lower limit to the shape parameter $(\gamma=1)$ is dictated by the regime in which a single displacement is sufficient to fully decorrelate the signal. The variation of $\gamma$ predicted by this model is manifestly more limited than what we observe. It is possible however that a similar model may hold in our case, if a spatially heterogeneous distribution of relaxation processes generates a distribution of relaxation times, leading to $\gamma<1$. On the other hand, the upper limit of the range $(\gamma=1.5)$ predicted by this model is connected with the power law decay of the probability distribution function of the displacements $\Delta R$ observed in the gel. This distribution is assumed to be Lévy-like $[39,40]$ with its tail towards large $\Delta R$ being proportional to $\Delta R^{-p}$ with $p=\gamma+1=2.5$. Therefore, our results $(\gamma=1.8$ at low $q$ and high concentration) would imply a steeper decay of the displacements' probability distribution in the low- $q$ and large- $\Phi$ regime, leading to an increased ballisticlike character of the dynamics.

Encouraged by these considerations, we compare our results with a variant of the model proposed in [39]; the values of $\gamma$ measured at each concentration are then plotted in Fig. 7(a) on an adimensional axis provided by the scaled scattering vector $q_{\|} \delta$ so that they overlap, collapsing on a master curve, in analogy with Fig. 3 of [39]. In this construction, an overall scale factor needs to be determined. This is accomplished by noting that for $q_{\|} \delta=2$ the model predicts $\gamma=1$; therefore the absolute values of $\delta$ can be determined. The master curve thus obtained is in agreement with the model curve up to $q_{\|} \delta=2$, above which value the model would plainly predict $\gamma=1$, while we observe a stretched shape. As anticipated, this can be reconciled with the model if we assume that in our case we observe a spatially heterogeneous distribution of relaxation times. The scaling parameter $\delta$, reported as a function of $\Phi$
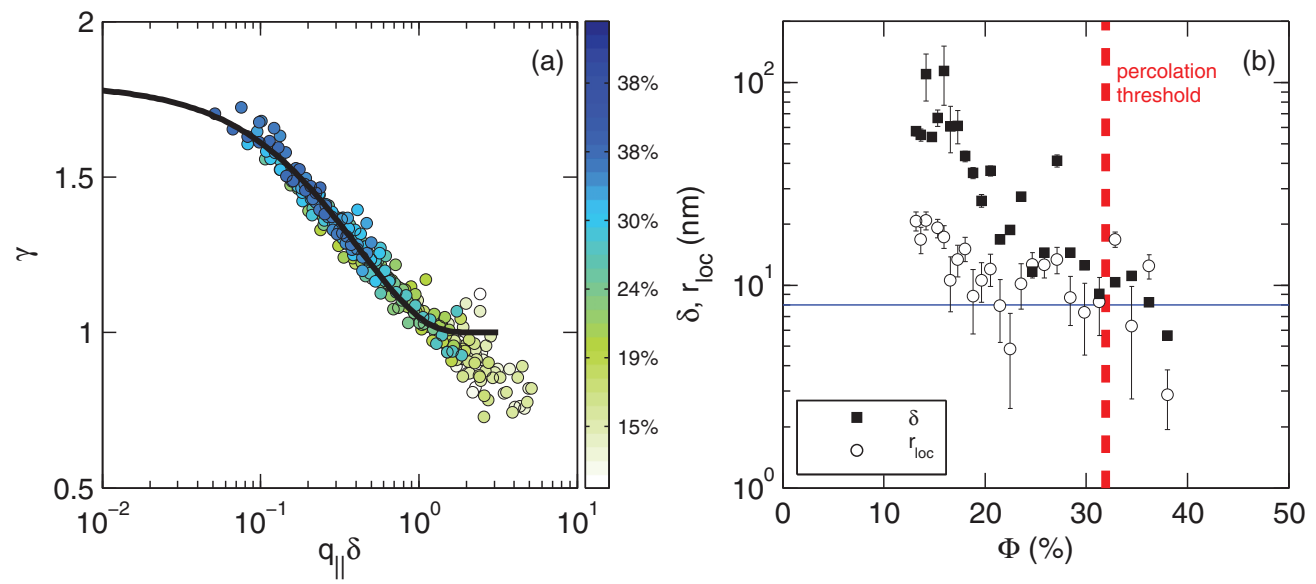

FIG. 7. (Color online) Master curve built from $\gamma$ as a function of the scaled scattering vector at different $\Phi$. (a) $\gamma\left(q_{\|}\right)$curves are shifted along the $x$ axis by the scaling length $\delta$ so that they overlap. The resulting master curve is in partial agreement with the model (solid line) proposed by Duri and Cipelletti [39]. (b) Long-time displacement length $\delta$ as a function of $\Phi$, compared with the short-time localization length $r_{\text {loc }} . \quad \delta$ becomes comparable with the particle size $\left(8 \mathrm{~nm}\right.$, horizontal line) and $r_{\text {loc }}$ above the percolation threshold (vertical dashed line). 


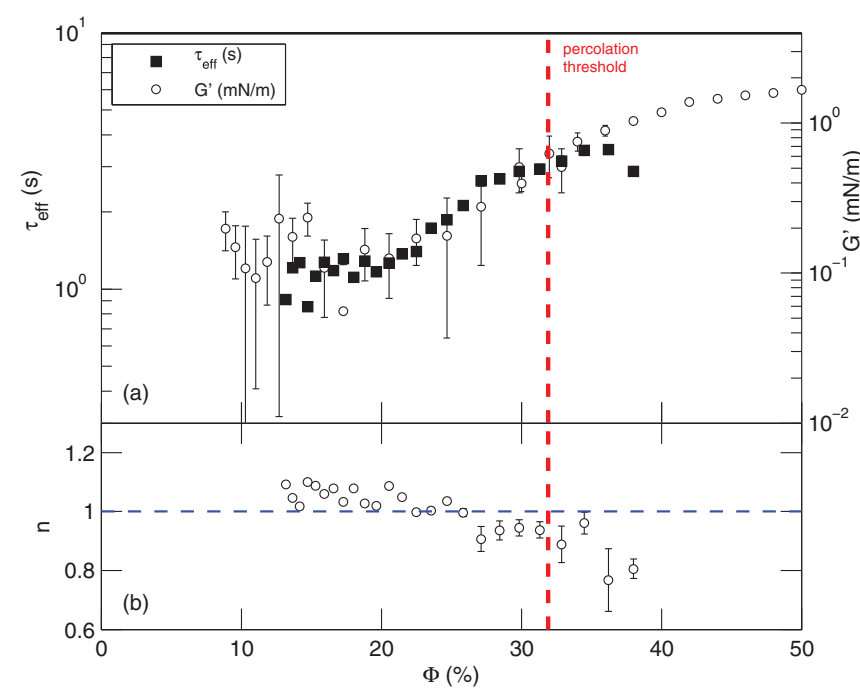

FIG. 8. (Color online) Approaching the percolation transition, the shear modulus $G^{\prime}$ and the relaxation time $\tau$ increase following the same law. (a) Comparison of $G^{\prime}$ and $\tau$ as functions of $\Phi$ : they follow the same behavior. Limit values are reached by both quantities above the percolation threshold (vertical red line). (b) The parameter $n$ in the dependence $\tau \propto q_{\|}^{-n}$ decreases as the concentration is increased. The blue dashed line represents $n=1$.

in Fig. 7(b), naturally offers us an estimate of the "long-time displacement length," i.e., of the dynamics taking place on the time scale of $\tau$. In the same figure, it is compared with the "short-time localization length" $r_{\text {loc }}$, deduced from the Debye-Waller decay of the contrast, which on the contrary characterizes the length scale of the fast dynamics. While it is notable that a single framework is able to describe the dynamics of this system over a broad range of concentrations, detailed inspection of the figure suggests that two different regimes exist, above and below the percolation threshold: below it, the length scales $r_{\text {loc }}$ and $\delta$ of the fast and slow dynamics decouple, while above it they become equal within experimental accuracy, and also comparable to the size of a single nanoparticle.

Figure 8 reports the concentration dependence of $\tau$ and of the exponent $n$ detailing the dependence of $\tau$ on $q_{\|}$. Defining the "effective" relaxation time as $\tau_{\text {eff }}=\tau \Gamma(1 / \gamma) / \gamma$ [41] (where $\Gamma$ is the Gamma function) we can compare its behavior with that of the mechanical modulus $G$. This is shown, for $q_{\|}=$ $0.015 \mathrm{~nm}^{-1}$, in Fig. 8(a); $\tau_{\text {eff }}$ increases as the concentration is raised in the same fashion as the elastic shear modulus $G$ '.

In the high-concentration regime (e.g., around the percolation threshold) this finding is consistent with the prediction of the already mentioned Bouchaud-Pitard model [38] for the relaxation in an elastic solid. In this case, $\tau$ is predicted to be proportional to the elastic modulus and inversely proportional to $q$ as observed experimentally and reported in Fig. 8. We recall, however, how the model partly failed in explaining the compressed-to-stretched transition of the correlation functions' shape reported in Fig. 5(c) and discussed before.

Notably, we find that the agreement between $\tau_{\text {eff }}$ and $G$ holds over the whole $\Phi$ range, even at those lower concentra- tions where the correlation function shape is stretched, namely, for $\Phi<18 \%$. This may be rationalized by considering that, even in this regime, the film's dominant mechanical response is elastic. In a similar nanogel in 3D, Guo et al. [42] verified a relation between $G^{\prime}$ and the localization length which is postulated in a self-consistent mode-coupling theory [43]: this relation does not hold in our case, as $\tau$ is constant for $\Phi<18 \%$ within the error, while the localization length [Fig. 4(b)] decreases. The general picture emerging from the agreement between $\tau_{\text {eff }}$ and $G^{\prime}$ is that of a system in which fluctuation-dissipation relations hold, even connecting results spanning very different space scales, from microns (GI-XPCS) to centimeters (rheometry).

Figure 7(b) reports the dependence of the exponent $n$ on $\Phi$. The coefficient $n \approx 1$ in the scaling $\tau \propto q_{\|}^{-n}$ observed by us in the stretched regime $(\Phi<18 \%)$ is unexpected and, to the best of our knowledge, it has been reported before only in the case of Laponite. Further theoretical investigations may be needed in order to formulate a physical model for this behavior. On the contrary, the slight reduction of the exponent $n$ towards 0.8 observed in the compressed regime is consistent with what was already observed at higher concentration in the same system [18].

\section{Dynamics and relaxation following a perturbation}

Following the characterization of the spontaneous dynamics of the $2 D$ network as a function of $\Phi$, we now focus on the effects on the dynamics caused by an external mechanical perturbation due to the oscillatory movement of a magnetic needle floating on the surface, in a similar fashion to the ISR apparatus [24] used to measure the mechanical modulus of the system, as better described in the experimental section. To check for stationarity, and to better characterize each starting point, the dynamics of the system was measured before applying any perturbation; then the perturbation was applied for $10 \mathrm{~min}$, with the magnetic needle put into angular oscillation on the surface, at a frequency of $10 \mathrm{~Hz}$ and amplitude $\alpha \approx 5^{\circ}$. The needle was $4 \mathrm{~cm}$ long; given the total area occupied by the film, this perturbation induced a relative area variation $d A / A \approx 0.3 \%$. The perturbation ended by bringing the needle back to its initial position. In this way, we can exclude the possibility that the dynamics observed after the perturbation reflects some obvious stress relaxations on the macroscale.

During the needle's movement, no GI-XPCS measurement was possible, due to the obvious and drastic decorrelation induced by the mechanical perturbation. After switching off the perturbation, the dynamics of the network was measured following the usual procedure, mapping the evolution of the dynamical parameters extracted from the correlation functions as a function of the experimental time, on a temporal scale of tens of minutes.

The experiment was performed at two different concentrations, namely, at $\Phi=9 \%$ and $\Phi=36 \%$, chosen to be below and above the percolation threshold; the results are reported in Figs. 9(a) and 9(b). The first, striking result is that in both cases the localization length $r_{\text {loc }}$ is found to be noticeably reduced by the perturbation, and its unperturbed value is recovered only on a time scale as slow as 10-20 min, although the recovery seems 

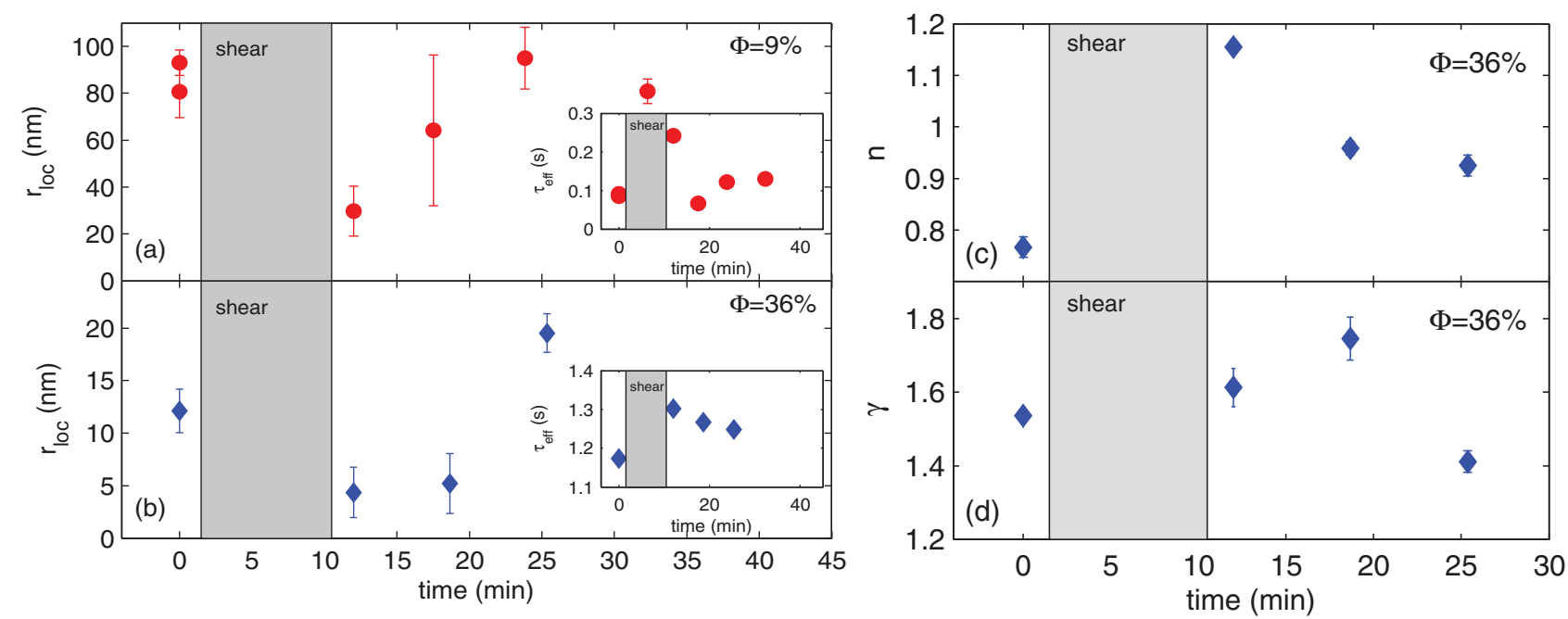

FIG. 9. (Color online) A mechanical perturbation affects the fluctuation dynamics in different ways for films above and below the percolation threshold. The localization length, measured below [9\% (a)] and above [36\% (b)] the percolation threshold. While a major effect of compaction is induced at $9 \%$, very little effect is produced at $36 \%$; alongside this, the recovery of the unperturbed state is much quicker at low concentration. Insets: Effective relaxation times increase after perturbation, measured at $q_{\|}=0.02 \mathrm{~nm}^{-1}$.(c),(d) At $\Phi=36 \%$, the mechanical perturbation causes an increase in the slope of the $\tau \propto q_{\|}^{-n}$ dependence and in the shape exponent $\gamma$.

to be faster at low concentration. Alongside this, $\tau$ is increased; at concentration $\Phi=9 \%$ it relaxes back to values close to those of the system's unperturbed state in less than $10 \mathrm{~min}$, while at concentration $\Phi=36 \%$, the effect of the perturbation on the dynamics is persistent on a much longer time scale, indicating that a relevant permanent modification of the $2 \mathrm{D}$ network might have been induced. Consistently, for $\Phi=36 \%$, the parameter $n$ shows an increase from $n \simeq 0.8$ (unperturbed value) up to $n \simeq 1.2$ immediately after the perturbation, to slowly decrease at subsequent times towards the unperturbed value [Fig. 9(c)].

More relevant changes are found in the analysis of the shape parameter [Fig. 9(d)]. The perturbation induces a more compressed form, reaching $\gamma \simeq 1.8$ at $q_{\|}=0.02 \mathrm{~nm}^{-1}$, consistent with the interpretation of the data provided in [43] for Laponite. Notably, this stress-induced effect was not observed instantaneously at the switching off of the perturbation; rather it peaks roughly $10 \mathrm{~min}$ after this. For longer times, the system then reaches a new state characterized by $\gamma \simeq 1.4$, a value slightly lower than the unperturbed state, which may be interpreted as a new stationary state in which stress relaxation is less important than before the perturbation was applied.

In order to investigate the temporal evolution of the dynamics and to shed more light on transient effects, we calculate the two-time correlation functions, defined as

$$
C\left(t_{1}, t_{2}\right)=\frac{\left\langle I\left(t_{1}\right) I\left(t_{2}\right)\right\rangle}{\left\langle I\left(t_{1}\right)\right\rangle\left\langle I\left(t_{2}\right)\right\rangle}
$$

where the average is performed over the pixels characterized by the same $q_{\|}$and $I\left(t_{1}\right)$ and $I\left(t_{2}\right)$ are the scattered intensities measured at two different experimental times $t_{1}$ and $t_{2}$. It is customary to represent it as a function of the aging time $t_{\text {age }}=$ $\left|t_{2}+t_{1}\right| / 2$ and of the lag time $\tilde{\tau}=\left|t_{2}-t_{1}\right|$; graphically, one can imagine extracting the $g^{(2)}\left(t_{\mathrm{age}}, \tilde{\tau}\right)$ correlation functions by selecting rectangular slices of $C\left(t_{1}, t_{2}\right)$ taken perpendicular to its main diagonal [14].
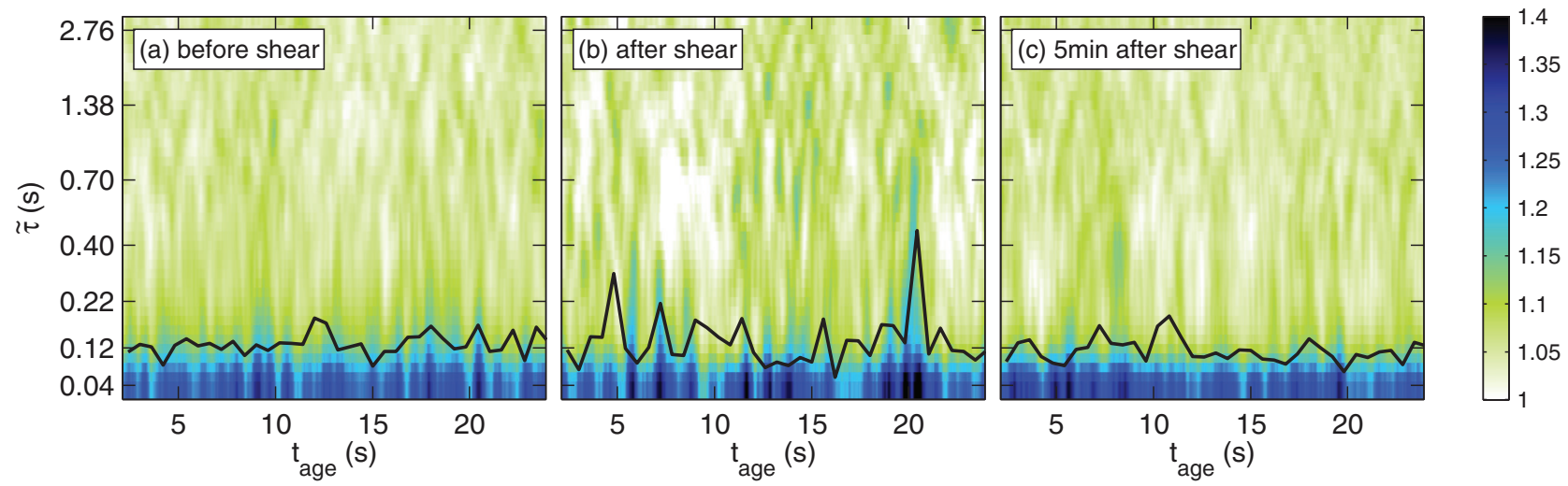

FIG. 10. (Color online) Insight into the effects of the mechanical stress on the dynamics, displaying the age dependence of the correlation functions $g^{(2)}\left(t_{\text {age }} ; \tilde{\tau}\right)$ at $\phi=9 \%$ and $q_{\|}=0.02 \mathrm{~nm}^{-1}$. Black lines represent $\tau$ as a function of the aging time. The mechanical stress induces heterogeneities in the characteristic time of the dynamics that disappear in a few minutes. 
The calculation is performed on data measured before and after the application of the stress, $q=0.02 \mathrm{~nm}^{-1}$ and for concentration $\Phi=9 \%$. Subsequently, the correlation functions $\left.g^{(2)}(\tilde{\tau})\right|_{\text {age }}$ selected at different values of $t_{\text {age }}$ were fitted with simple exponential decays, in order to obtain the evolution of the relaxation time $\tau$ with increasing $t_{\text {age }}$.

Figure 10 shows $g^{(2)}\left(t_{\text {age }} ; \tilde{\tau}\right)$ measured before and after the mechanical perturbation; the relaxation times $\tau\left(t_{\text {age }}\right)$ resulting from the fitting procedure are plotted as black lines. The application of the mechanical stress causes the onset of a dynamical heterogeneous state, with successive random appearance of slow dynamics, even after the mechanical perturbation has been switched off. This causes, on average, the slower relaxation time extracted from the correlation functions $g^{(2)}(\tau)$, reported in the inset of Fig. 9(a). The dynamical heterogeneous state disappears after a few minutes, with complete recovery of the unperturbed state.

This is confirmed by an analysis of the variance $\chi(\tilde{\tau})$ of the two-time correlation function, defined as

$$
\chi(\widetilde{\tau})=\left\langle C^{2}\left(t_{\mathrm{age}} ; \tilde{\tau}\right)\right\rangle-\left\langle C\left(t_{\mathrm{age}} ; \tilde{\tau}\right)\right\rangle^{2},
$$

which is also related to the dynamical susceptibility.

As shown in Fig. 11, in the unperturbed state the system is characterized by a featureless variance; after the mechanical perturbation, the variance shows a peak centered at $\tilde{\tau} \approx 0.8 \mathrm{~s}$, which disappears at longer times, when the effects of the perturbation vanish. The presence of a peak in the dynamical susceptibility is commonly associated, in the literature, with the onset of dynamical heterogeneity; in our case, it may indicate that the mechanical perturbation affects the 2D network in a nonuniform way, activating random relaxing stresses that propagate as density fluctuations and disappear in the time range of a few minutes. This phenomenology is consistent with analogous results obtained by means of DLS measurements on colloidal gel [39].

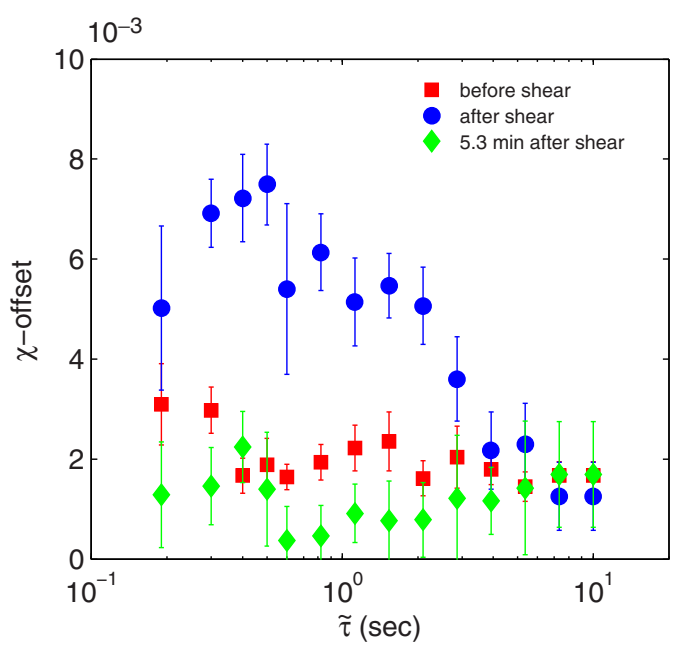

FIG. 11. (Color online) Effects of the mechanical stress on the variance $\chi$ of the two-time correlation functions, at $\phi=9 \%$. The mechanical stress induces an increase of the variance of the twotime correlation functions, with the appearance of a peak. This peak disappears after a waiting time of a few minutes (flat variance).

\section{CONCLUSIONS}

The evolution of the dynamics of a bidimensional gel formed by gold nanoparticles at the air/water interface has been characterized by means of GI-XPCS, supported by imaging and by rheology measurements. Mechanical measurements have shown that the structure behaves as a bidimensional elastic solid at all concentrations. The increase of the elastic shear modulus as a function of surface concentration closely mimics that of the GI-XPCS relaxation time. The general picture emerging is that of a system in which fluctuationdissipation relations hold, even connecting results spanning very different space scales, from microns (GI-XPCS) to centimeters (rheometry). In particular, this was predicted within a model proposed by Bouchaud and Pitard [38], which, however, fails to account for the observed shapes of the correlation functions; we document in our system an actively controlled transition from a stretched to a compressed shape. The observed behavior seems better reconcilable with a phenomenological model proposed by Duri and Cipelletti [39] which hypothesizes that the correlation of the scattered intensity is driven by rare intermittent rearrangements. Exploiting the unique opportunity offered by the Langmuir 2D geometry to continuously vary the concentration on the very same gel system, we could push this analysis further, and we have built a master curve for the shape parameter as a function of the reduced scattering vector. According to the model [39], the scaling factor of this master curve corresponds to the long-time displacement length $\delta$, characterizing the dynamics happening on the time scale of a few seconds. This is distinct from the short-time localization length $r_{\text {loc }}$, determined by the usual pseudo-Debye-Waller analysis of the initial contrast, which characterizes dynamics faster than $10 \mathrm{~ms}$. In the low-concentration regime we find the $\delta \simeq 100 \mathrm{~nm}$, much larger than the value of $r_{\text {loc }}$; however, as the concentration $\Phi$ increases towards the percolation threshold, the two lengths converge to a common limit comparable to the single-particle size.

We also address dynamical heterogeneities, extending our previous observations [18] by focusing here on the low-concentration and the deeply out-of-equilibrium regimes induced by an external mechanical perturbation. This perturbation induces an increase of the relaxation time, accompanied by an increase of the shape exponent $\gamma$. This fact indicates a more compressed, ballisticlike character of the dynamics as a consequence of the perturbation applied, and suggests an analogy with the results obtained by XPCS on aged and rejuvenated Laponite [43].

At the same time, perturbation enhances the temporal heterogeneities, leading to the appearance of a peak in the variance. After the end of the perturbation, the relaxation towards the initial stationary state happens on the time scale of a few minutes at low concentration, while at high concentration it takes much longer.

\section{ACKNOWLEDGMENTS}

The ESRF is gratefully acknowledged for provision of beam time; Oleg Konovalov (ESRF) for access to the Langmuir laboratory; Karim Lhoste (ESRF) for technical support; Pietro 
Cicuta (Cambridge University, U.K.) for useful discussions on the formation of the 2D gel. This work is partly held under the umbrella of the COST CM1101 and MP1106 Actions.
[1] R. Bhattacharya and J. K. Basu, J. Colloid Interface Sci. 396, 69 (2013).

[2] K. Larson-Smith and D. Pozzo, Langmuir 28, 11725 (2012).

[3] C. P. Collier, Science. 277, 1978 (1997).

[4] J. Huang, F. Kim, A. R. Tao, S. Connor, and P. Yang, Nat. Mater. 4, 896 (2005).

[5] J. He, X.-M. Lin, H. Chan, L. Vuković, P. Kraál, and H. M. Jaeger, Nano Lett. 11, 2430 (2011).

[6] K. E. Mueggenburg, X.-M. Lin, R. H. Goldsmith, and H. M. Jaeger, Nat. Mater. 6, 656 (2007).

[7] F. Bresme and M. Oettel, J. Phys.: Condens. Matter 19, 413101 (2007).

[8] B. D. Leahy, L. Pocivavsek, M. Meron, K. L. Lam, D. Salas, P. J. Viccaro, K. Y. C. Lee, and B. Lin, Phys. Rev. Lett. 105, 058301 (2010).

[9] D.-M. Smilgies, A. T. Heitsch, and B. a Korgel, J. Phys. Chem. B 116, 6017 (2012).

[10] K. Vegso, P. Siffalovic, E. Majkova, M. Jergel, M. Benkovicova, T. Kocsis, M. Weis, S. Luby, K. Nygård, and O. Konovalov, Langmuir 28, 10409 (2012).

[11] D. C. E. Calzolari, D. Pontoni, M. Deutsch, H. Reichert, and J. Daillant, Soft Matter 8, 11478 (2012).

[12] L. Isa, D. C. E. Calzolari, D. Pontoni, T. Gillich, A. Nelson, R. Zirbs, A. Sánchez-Ferrer, R. Mezzenga, and E. Reimhult, Soft Matter 9, 3789 (2013).

[13] O. Dauchot, D. J. Durian, and M. van Hecke, in Dynamical Heterogeneities in Glasses, Colloids, and Granular Media, edited by L. Berthier, G. Biroli, J.-P. Bouchaud, L. Cipelletti, and W. VanSaarloos (Oxford Scholarship Online, Oxford, U.K., 2011).

[14] A. Madsen, R. L. Leheny, H. Guo, M. Sprung, and O. Czakkel, New J. Phys. 12, 055001 (2010).

[15] D. El Masri, L. Berthier, and L. Cipelletti, Phys. Rev. E 82, 031503 (2010).

[16] L. Cristofolini, Curr. Opin. Colloid Interface Sci., doi:10.1016/j.cocis.2014.03.006 (2014).

[17] D. Orsi, L. Cristofolini, M. P. Fontana, E. Pontecorvo, C. Caronna, A. Fluerasu, F. Zontone, and A. Madsen, Phys. Rev. E 82, 031804 (2010).

[18] D. Orsi, L. Cristofolini, G. Baldi, and A. Madsen, Phys. Rev. Lett. 108, 105701 (2012).

[19] D. Orsi, G. Baldi, P. Cicuta, and L. Cristofolini, Colloids Surf. A 413, 71 (2012).

[20] H. Schmidle, S. Jäger, C. K. Hall, O. D. Velev, and S. H. L. Klapp, Soft Matter 9, 2518 (2013).

[21] D. Orsi, A. Vezzani, R. Burioni, A. Pucci, G. Ruggeri, and L. Cristofolini, Colloids Surfaces A 441, 912 (2014).
[22] L. Onsager and S. Machlup, Phys. Rev. 91, 1505 (1953).

[23] P. Cicuta and E. M. Terentjev, Eur. Phys. J. E: Soft Matter Biol. Phys. 16, 147 (2005).

[24] C. F. Brooks, G. G. Fuller, C. W. Frank, and C. R. Robertson, Langmuir 15, 2450 (1999).

[25] D. Orsi, L. Cristofolini, and M. P. Fontana, J. Non-Cryst. Solids 357, 580 (2011).

[26] R. L. Leheny, Curr. Opin. Colloid Interface Sci. 17, 3 (2012).

[27] G. Grübel, A. Madsen, and A. Robert, in Soft Matter Characterization, edited by R. Borsali and R. Pecora (Springer, Berlin, 2008), pp. 935-995.

[28] S. Q. Choi, S. Steltenkamp, J. A. Zasadzinski, and T. M. Squires, Nat. Commun. 2, 312 (2011).

[29] M. Brust, M. Walker, D. Bethell, D. J. Schiffrin, and R. Whyman, J. Chem. Soc. Chem. Commun. 801 (1994).

[30] A. Pucci, N. Tirelli, E. A. Willneff, S. L. M. Schroeder, F. Galembeck, and G. Ruggeri, J. Mater. Chem. 14, 3495 (2004).

[31] M. Dionisio, F. Maffei, E. Rampazzo, L. Prodi, A. Pucci, G. Ruggeri, and E. Dalcanale, Chem. Commun. (Cambridge) 47, 6596 (2011).

[32] J. T. Petkov, T. D. Gurkov, B. E. Campbell, and R. P. Borwankar, Langmuir 16, 3703 (2000).

[33] C. Ponchut, J. M. Rigal, J. Clément, E. Papillon, A. Homs, and S. Petitdemange, J. Instrum. 6, C01069 (2011).

[34] B. J. Berne and R. Pecora, Dynamic Light Scattering (Dover, New York, 2000).

[35] C. Caronna, Y. Chushkin, A. Madsen, and A. Cupane, Phys. Rev. Lett. 100, 055702 (2008).

[36] B. Ruta, Y. Chushkin, G. Monaco, L. Cipelletti, E. Pineda, P. Bruna, V. M. Giordano, and M. Gonzalez-Silveira, Phys. Rev. Lett. 109, 165701 (2012).

[37] R. Angelini, L. Zulian, A. Fluerasu, A. Madsen, G. Ruocco, and B. Ruzicka, Soft Matter 9, 10955 (2013).

[38] J.-P. Bouchaud and E. Pitard, Eur. Phys. J. E: Soft Matter Biol. Phys. 6, 231 (2001).

[39] A. Duri and L. Cipelletti, Europhys. Lett. 76, 972 (2006).

[40] L. Cipelletti, L. Ramos, S. Manley, E. Pitard, D. A. Weitz, E. E. Pashkovski, and M. Johansson, Faraday Discuss. 123, 237 (2003).

[41] H. Guo, G. Bourret, R. B. Lennox, M. Sutton, J. L. Harden, and R. L. Leheny, Phys. Rev. Lett. 109, 055901 (2012).

[42] H. Guo, S. Ramakrishnan, J. L. Harden, and R. L. Leheny, Phys. Rev. E 81, 050401(R) (2010).

[43] K. S. Schweizer and G. Yatsenko, J. Chem. Phys. 127, 164505 (2007). 